ISSN: 2386-3919 - e-ISSN: 2386-3927

DOI: https://doi.org/10.14201/et20173513963

\title{
ACTITUDES DOCENTES HACIA LA INTEGRACIÓN CURRICULAR DE LA PRENSA DIGITAL EN LOS CENTROS ESCOLARES DE EDUCACIÓN OBLIGATORIA
}

\author{
Teacher attitudes towards curriculum integration of digital \\ press in school centers of Compulsory Education
}

\author{
Inmaculada MARTÍN HERRERA \\ Centro Universitario San Isidoro.Correo-e: imartin@ceade.es
}

Recibido: 08/02/2016; Aceptado: 15/02/2017; Publicado: 31/05/2017

Ref. Bibl. INMACULADA MARTÍN HERRERA. Actitudes docentes hacia la integración curricular de la prensa digital en los centros escolares de Educación Obligatoria. Enseñanza \& Teaching, 35, 1-2017, 39-63.

RESUMEN: Con este estudio pretendemos conocer la actitud del profesorado hacia la integración curricular de la prensa digital en los centros escolares de Educación Obligatoria. La investigación es de corte descriptivo, pues nos interesa evaluar las percepciones docentes acerca del potencial educativo de la ciberprensa. Además de averiguar cómo influyen en estas opiniones una serie de variables personales, académicas e institucionales. La muestra está integrada por 601 docentes, y los datos se han recopilado mediante un cuestionario de elaboración propia que consta de ocho preguntas abiertas con las que se ha llevado a cabo un análisis de contenido, empleando para ello el programa Atlas.ti.7. Los resultados concluyen que la mayoría del profesorado tiene una opinión favorable sobre la ciberprensa, y la considera un material que puede emplearse como herramienta didáctica en prácticamente todas las asignaturas de la Educación Obligatoria, y para un amplio y variado espectro de actividades. Los docentes perciben que la capacidad crítica y orientadora y el espíritu autodidacta son algunas de sus habilidades para educar con estos medios. Aunque también detectan carencias, especialmente en lo que se refiere a la falta de recursos tecnológicos en las escuelas y la necesidad de planes de formación específicos en el manejo de plataformas digitales. Finalmente, atendiendo a las implicaciones del 
INMACULADA MARTÍN HERRERA

ACTITUDES DOCENTES HACIA LA INTEGRACIÓN CURRICULAR DE LA PRENSA DIGITAL

EN LOS CENTROS ESCOLARES DE EDUCACIÓN OBLIGATORIA

estudio, este diagnóstico sobre la relación actual entre el centro escolar y la ciberprensa se plantea como un marco de referencia para la puesta en marcha de acciones formativas que permitan una enseñanza de calidad por y para los medios en la Red.

Palabras clave: internet; medios de comunicación; actitudes docentes; alfabetización mediática; Educación Obligatoria.

SUMMARY: With this study we aim to know the attitude of the teaching staff towards curriculum integration of digital press in school centers of Compulsory Education, as part of a process of media and digital literacy necessary for communication and teaching in the XXI century. This research presents a descriptive approach. We are also interested in finding out how a series of personal, academic and institutional variables influence the opinions of teaching staff. With respect to the sample, it is made up of 601 teachers, and data has been compiled through our own questionnaire with eight open questions; out of which a content analysis has been carried out using the software Atlas.ti 7. Results conclude that most of teaching staff has a favourable opinion of digital press and it is considered a material that can be used as a teaching tool almost in every subject of Compulsory Education and for a wide and varied range of activities. Teachers see that the critical and guiding ability and the self-taught spirit are some of the advantages to teach with these means. Although some weak points are also identified, especially referred to the lack of technological resources available in schools and the need for specific training schemes in the use of digital platforms. Finally, answering to the study implications, this diagnosis about the current relation between the school center and the digital press is presented as a reference framework for the start-up of teaching actions that allow for a quality media education in the Net.

Key words: internet; mass media; teacher attitudes; media education; Compulsory Education.

\section{INTRODUCCIÓN}

Esta investigación se lleva a cabo con objeto de conocer, a través de las opiniones y actitudes del profesorado de Educación Obligatoria, el papel que le corresponde a la educación formal en la integración curricular de la prensa online. Actualmente, es menester que la escuela eduque para la sociedad informacional y tecnológica, y colabore de manera conjunta con los medios de comunicación en un necesario proceso de alfabetización múltiple: audiovisual, mediática y digital. De manera que los mensajes periodísticos y las Tic se usen y se consuman de manera consciente, teniendo en cuenta su enorme influencia sobre la manera de pensar y de obrar de los menores. Para Snyder (2016: 313), «una educación para la ciudadanía digital es una necesidad mundial»; así como un desafío para las administraciones, los profesores, los estudiantes y los padres que deben solicitar la reforma educativa que promueva la integración 
ACTITUDES DOCENTES HACIA LA INTEGRACIÓN CURRICULAR DE LA PRENSA DIGITAL EN LOS CENTROS ESCOLARES DE EDUCACIÓN OBLIGATORIA

de estos medios en las escuelas para garantizar su uso responsable y seguro en beneficio de la sociedad global.

Respecto a la justificación del estudio, consideramos que conocer previamente la relación que mantiene el centro escolar con la ciberprensa es fundamental para el desarrollo y la implantación de actuaciones docentes que permitan hacer frente a los continuos cambios que se producen en el actual entorno educativo y comunicacional del que todos participamos. Máxime cuando hablamos de alumnos que son nativos digitales (Prensky, 2001) y, por tanto, personas que «están creciendo y educándose con la presencia de Internet en los principales espacios de socialización» (Marí, 2006: 113).

\section{BREVE FUNDAMENTACIÓN}

La fundamentación teórica de esta investigación se sustenta sobre tres grandes temas: la tecnología educativa, y más concretamente aquella que hace referencia a la prensa digital como herramienta didáctica; las relaciones entre el centro escolar y la ciberprensa; y el profesorado, pues nuestro trabajo es, precisamente, un estudio de percepciones docentes.

\subsection{Usos didácticos de la prensa digital en la Educación Obligatoria}

En relación a la tecnología educativa, nos hemos centrado en destacar las ventajas y los beneficios del uso de la prensa digital como instrumento educativo en las diferentes asignaturas de la Educación Obligatoria. La literatura consultada (Amar, 2010; Ballesta y Bernal, 2013; Buil, Hernández y Sesé, 2013; Feijoo, 2015; González Prieto, 2010; López y Aguaded, 2015; Moore, 2013; Pinilla, 2008; Said, 2010) nos ha servido de inspiración para proponer las siguientes aplicaciones de la prensa online con fines didácticos en los centros escolares: servir de apoyo y complemento a los materiales curriculares; realizar actividades interactivas; fomentar la lectura y el aprendizaje de vocabulario; proporcionar temas para el debate, lo que contribuye a crear una clase democrática, conectada con la realidad y más participativa; practicar con las características de los textos periodísticos en la Red; aprender a leer el lenguaje hipertextual; contrastar las noticias; comparar los mismos contenidos en distintos periódicos; participar en el proceso informativo mediante la publicación de comentarios propios; mejorar la expresión oral y escrita; distinguir los contenidos periodísticos de la publicidad, etc.

También existen aspectos negativos que debemos tener en cuenta. En este sentido, y atendiendo a las carencias tecnológicas presentes en algunas escuelas, una de las desventajas que conlleva trabajar en clase con estos soportes es la necesidad de disponer de los equipos y los programas informáticos adecuados. Además, la dedicación del docente debe ser intensiva, pues al mismo tiempo que educa a sus alumnos en medios digitales, debe controlarlos para que usen el 
ordenador convenientemente, respeten la propiedad intelectual y no accedan a contenidos inapropiados.

\subsection{Relaciones entre el centro escolar y la ciberprensa}

Sobre las relaciones entre el centro escolar y la prensa digital, la revisión bibliográfica nos ha permitido deducir que, en general, el profesorado apoya la integración escolar de las publicaciones online porque estos periódicos son la mejor manera de aprender sobre el mundo (Little, 2014); optimizan el proceso de aprendizaje (Buil et al., 2013); permiten «aplicaciones en el campo de la comunicación educativa y una mayor innovación pedagógica" (Cebrián Herreros, 2009: 10); y contribuyen al desarrollo cognitivo de los alumnos (Mejía y Acero, 2015). Por todo ello, debemos aprovechar su potencial para desarrollar líneas de reflexión (Morduchowicz, 2012) y "plantearnos metodologías colaborativas que nos ayuden a favorecer una educación en y para el consumo de medios digitales» (Ballesta, Cerezo y Veas, 2014: 22).

Alonso, Ospina y Sánchez (2014) también apuestan por su uso didáctico, con un propósito pedagógico bien definido en la educación básica, media y superior, y como un recurso interdisciplinar que permite, mediante su transversalidad, integrar las distintas áreas del conocimiento en los procesos curriculares. Por su parte, desde un enfoque eminentemente práctico y realista, Player-Koro (2013) considera que al trabajar con estas plataformas tecnológicas hay que distanciarse de la retórica entusiasta tradicional y contribuir a discusiones más realistas. Por tanto, según nuestro criterio, la postura más adecuada es la aceptación crítica; es decir, un equilibrio que asume las posibilidades de los cibermedios y, también, sus limitaciones educativas.

\subsection{El profesor como orientador y modelo de conducta}

Por último, la tercera fuente teórica está dedicada a los profesores, donde hemos podido constatar que dentro del ámbito de la Didáctica y de la Organización Escolar se ha concedido mucha importancia al paradigma mediacional focalizado en el docente. Nos referimos a metodologías que se centran en el proceso cognitivo del alumno, siendo el profesor un guía y asesor (Cabero et al., 2011; Delors, 1996; Pedrosa, 2010; Prensky, 2014; Unesco, 2008) que influye para que el estudiante piense y actúe como un sujeto activo durante su aprendizaje con la ciberprensa. Pues, tal y como apunta López Gil (2016), el acompañamiento a los estudiantes en los procesos de lectura digital es una realidad urgente de abordar en las instituciones educativas. De manera que atendiendo, a este rol de orientador, la integración de la educación en medios en el día a día de la escuela "debe involucrar a todo el profesorado y abarcar todos los temas de estudio" (Spanhel, 2011: 182). 
ACTITUDES DOCENTES HACIA LA INTEGRACIÓN CURRICULAR DE LA PRENSA DIGITAL EN LOS CENTROS ESCOLARES DE EDUCACIÓN OBLIGATORIA

En nuestra investigación, se ha elegido al profesorado como entidad valorativa del problema porque sus percepciones, conocimientos y actitudes en relación a estos medios se plasman en sus clases, e influyen de manera significativa en sus prácticas docentes y en la propia concepción del alumnado. Es decir, en lo que piensan y en lo que hacen estos niños y jóvenes cuando se informan a través de las publicaciones online. Por todo ello, los profesores son una pieza clave en la educación mediática a la hora de enseñar los usos correctos de la prensa digital.

\section{OBJETIVOS Y DISEÑO DE LA INVESTIGACIÓN}

Entendemos que para determinar el potencial educativo de la prensa digital es preciso conocer las percepciones del profesorado acerca de este medio de comunicación. Y en base a esta premisa, nuestro problema de investigación se ha concretado en dos objetivos principales: 1) Conocer las opiniones del profesorado de Educación Primaria y Educación Secundaria Obligatoria de Sevilla capital acerca de la integración y el empleo de la prensa digital en los centros escolares. 2) Conocer cómo influyen sobre estas opiniones una serie de variables personales, académicas e institucionales.

El diseño de la investigación se caracteriza por encuadrarse dentro de una metodología no experimental en la cual el científico no tiene control directo sobre las variables independientes, porque "ya acontecieron sus manifestaciones o por ser intrínsecamente no manipulables» (Kerlinger, 1975: 268). Se trata, igualmente, de un estudio descriptivo porque tratamos de describir y evaluar ciertas características de las percepciones de los docentes de Educación Obligatoria acerca del potencial educativo de la prensa digital.

\subsection{Población y muestra}

La población de este trabajo está compuesta por 7.437 profesores y profesoras de Educación Obligatoria de la ciudad de Sevilla. Conocida la población objeto de estudio se realizó la selección de la muestra (Gráfica 1), que está integrada por 601 docentes pertenecientes a 76 centros escolares, de los cuales 31 son colegios públicos de Educación Primaria (CEIP), 28 son institutos de Educación Secundaria (IES) y 17 son colegios privados concertados (CC). Se trata de una muestra representativa, que ha sido elegida mediante la fórmula de poblaciones finitas propuesta por Balestrini (1999); y en la que se ha trabajado con un nivel de confianza del 95,5\% y con un error muestral del 3,51\%. 
GRÁFICA 1

Muestra de la investigación

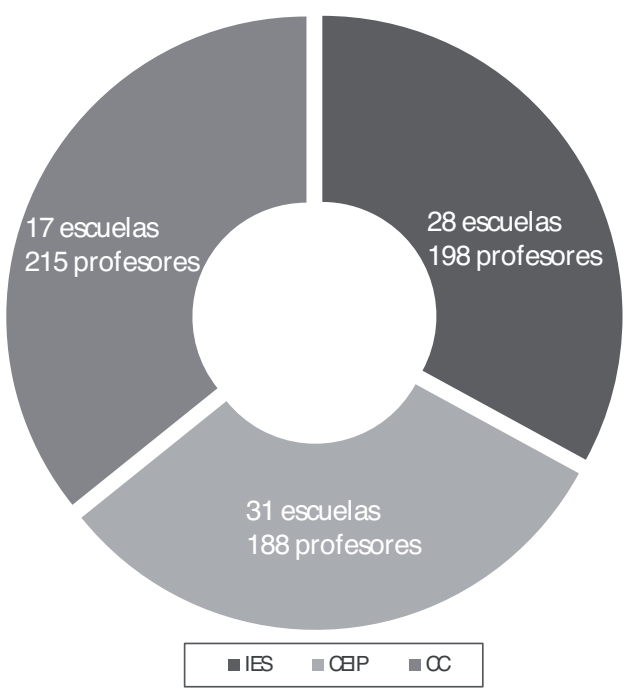

\subsection{Instrumento de medida}

Para la recogida de los datos durante la etapa de muestreo, que se llevó a cabo a lo largo del año 2015, se elaboró un cuestionario. Este instrumento de medida consta, en primer lugar, de un apartado donde, además de presentarle la investigación al destinatario, se recogen las características identificativas del docente mediante una serie de datos personales, académicos e institucionales. El siguiente bloque está integrado por ocho preguntas abiertas (Cuadro 1) con las que se realizó un análisis de contenido, definido por Krippendorff (1990) como un tipo de estudio que permite "formular, a partir de ciertos datos, inferencias reproducibles y válidas que pueden aplicarse a un contexto» (p. 28). 
ACTITUDES DOCENTES HACIA LA INTEGRACIÓN CURRICULAR DE LA PRENSA DIGITAL EN LOS CENTROS ESCOLARES DE EDUCACIÓN OBLIGATORIA

CUADRO 1

Preguntas abiertas del cuestionario

1. ¿Qué opina de la prensa electrónica?

2. ¿Cómo mejoraría la prensa digital?

3. En su labor docente, ¿en qué materias usaría la prensa electrónica?

4. ¿Y para qué tipo de actividades usaría la prensa electrónica?

5. ¿Cuáles son las capacidades que presentan los profesores a la hora de educar en medios de comunicación digitales?

6. ¿Cuáles son las carencias que presentan los profesores a la hora de educar en medios de comunicación digitales?

7. Según su opinión, ¿cómo influye el ambiente familiar en el uso que los niños y los adolescentes hacen de la prensa electrónica?

8. ¿Cómo puede influir el centro escolar en la utilización de la prensa digital por parte de sus alumnos?

El cuestionario fue validado mediante el juicio de siete expertos, quienes evaluaron el instrumento a partir de cuatro indicadores relativos a la coherencia de las preguntas con los objetivos de la investigación, así como a la claridad, la extensión y la adecuación del lenguaje de estos ítems a la muestra. Para la estimación de la fiabilidad se empleó la sentencia Reliability del programa estadístico spss 22, con el que se calculó el coeficiente de consistencia interna de Cronbach (Alfa de Cronbach), obteniéndose para todos los indicadores valores superiores a 0.8 . De manera que estos resultados pueden considerarse como una buena consistencia interna (Celina y Campo-Arias, 2005; George y Mallery, 2003), un grado de fiabilidad adecuado y, por lo tanto, se trata de un instrumento de medida fiable.

\subsection{Variables del estudio}

Uno de los objetivos del estudio era conocer cómo influyen sobre las opiniones del profesorado acerca de la prensa digital unas variables personales, académicas e institucionales. En este sentido, las variables estudiadas en esta investigación son, en primer lugar, las características personales género y edad. Existen antecedentes en la literatura científica (Ramírez, Cañedo y Clemente, 2012; Suárez, Almerich, Díaz y Fernández, 2012) acerca de la influencia de estas propiedades en investigaciones que pertenecen al ámbito de la tecnología educativa. Así pues, los estudios de estos autores concluyeron que los docentes hombres y los más jóvenes son los que se muestran más preparados y predispuestos a hacer prácticas con 
Internet. Y esto es así porque "han crecido en un contexto en el que las tecnologías digitales son parte consustancial de su vida diaria» (Suriá, 2011: 308).

Respecto a la variable académica especialidad: Primaria o Secundaria Obligatoria, consideramos que el nivel de estudios y las vivencias acumuladas en el trato con los alumnos en función de la etapa educativa son factores que inciden en las percepciones docentes, y por ello las hemos tenido en cuenta. Finalmente, elegimos la variable institucional tipo de centro: público o concertado, con la finalidad de conocer si la filosofía, la ideología religiosa o laica, así como las particularidades administrativas y de gestión derivadas de la titularidad del centro, como institución educativa y como empresa, influyen en los resultados del estudio.

\subsection{Análisis de los datos}

En este apartado describimos el proceso seguido para registrar la información y analizar los datos obtenidos mediante las preguntas abiertas que integran el cuestionario. Inspirándonos en Colás (1998), consideramos que el análisis de contenido que se lleva a cabo en este trabajo opera sobre textos. En nuestro caso, para construir los datos del estudio la información quedó recogida por escrito en el cuestionario. Cabe señalar que los textos se iban transcribiendo tan pronto como eran recibidos, e inmediatamente después se procedió a realizar el análisis de contenido.

Para la extracción de los datos definitivos, a los que llamamos unidades de registro o unidades de significación, y su posterior codificación, se ha empleado el programa informático Atlas.ti 7. En primer lugar, se elaboró una hoja Excel donde se introdujeron los textos y la información correspondiente a cada uno de los sujetos. Posteriormente, estos contenidos se importaron al citado programa Atlas.ti 7 , que actúa como un gestor de información. De manera que, una vez almacenados, organizados y etiquetados todos estos contenidos, fue posible conocer los resultados atendiendo a las variables.

Por tanto, el programa nos ayudó a analizar las redacciones con objeto de extraer el contenido conceptual de las mismas (Colás, 1998). Pues las respuestas de los profesores se han dividido o segmentado en unidades relevantes que, a su vez, mantienen una conexión semántica con el objetivo global de la cuestión. Lo que se pretende es que «el análisis no se limite a un tratamiento mecánico de los datos, sino que implique una actividad reflexiva, interpretativa y teórica de estos datos» (Colás, 1998: 289) para no perder la esencia subjetiva y personal que caracteriza a los mismos.

La recogida de estos datos o unidades de información implica que queden registrados por escrito en el archivo del programa Atlas.ti 7 creado para tal efecto. A continuación, desde el mismo programa, y siguiendo las indicaciones de Colás (1998), los datos segmentados fueron categorizados de acuerdo a un sistema organizado. Los códigos que aglutinan a estas unidades significativas pueden ser descriptivos, que son los que atribuyen un nombre a un contenido o segmento 
ACTITUDES DOCENTES HACIA LA INTEGRACIÓN CURRICULAR DE LA PRENSA DIGITAL EN LOS CENTROS ESCOLARES DE EDUCACIÓN OBLIGATORIA

del texto, por ejemplo 'mejoras'; o interpretativos, que identifican conceptos más abstractos, por ejemplo, 'familia-control', en referencia al control parental sobre el acceso de los hijos a Internet.

Estos temas se pueden identificar antes, durante y después de la recogida de los datos. En esta investigación se han empleado técnicas de codificación inductivas, pues no hemos tenido ninguna codificación previa a la recolección de los datos, sino que las categorías se han obtenido directamente de los datos. Se ha hecho así porque consideramos que, de esta manera, el material se amolda mejor a los códigos que lo representan, de modo que son códigos en uso y no códigos genéricos para muchos usos (Fernández, 2006).

Una vez establecidas las categorías y sus respectivas unidades de significación, procedimos a integrar la información y realizar el análisis de contenido a partir de los resultados. Dichos resultados se trasladaron de nuevo a un documento de Excel con objeto de obtener las tablas y las gráficas que permitieron la interpretación y la representación de los datos obtenidos. En concreto, este análisis se llevó a cabo mediante la triangulación de los datos, de manera que en cada una de las preguntas se estudiaron todas las categorías de respuesta a través del cálculo de las frecuencias de aparición y los porcentajes; y atendiendo a las variables consideradas, y que son: las características personales Género (masculino/femenino) y Edad (25 a 35 años/36 a 45 años/más de 46 años), la Especialidad Académica: Primaria o Secundaria Obligatoria y la característica institucional Tipo de Centro Escolar: público o concertado.

\section{PRESENTACión DE los RESUltados}

Tras el proceso de codificación obtuvimos varias categorías de respuesta para cada una de las preguntas. A su vez, estas etiquetas están integradas por un conjunto de ideas que se han extraído directamente de los textos proporcionados por el profesorado. Posteriormente, una vez realizado el análisis de contenido, donde se estudiaron todas las categorías mediante el cálculo de las frecuencias y los porcentajes, se obtuvieron los resultados que exponemos a continuación.

\section{1. ¿Qué opina de la prensa electrónica?}

Con esta pregunta pretendemos conocer qué opinión tiene el profesor o profesora sobre la prensa electrónica en cualquiera de sus facetas: medio de comunicación, agente social, herramienta educativa, etc. Dada la variedad de respuestas obtenidas, hubo que categorizar los textos quedando la distribución de las etiquetas y de sus correspondientes unidades de significación de la manera que se presenta en el Cuadro 2. 
INMACULADA MARTÍN HERRERA

ACTITUDES DOCENTES HACIA LA INTEGRACIÓN CURRICULAR DE LA PRENSA DIGITAL EN LOS CENTROS ESCOLARES DE EDUCACIÓN OBLIGATORIA

\section{CUADRO 2}

Codificación de las unidades de significación en la pregunta:

¿Qué opina de la prensa electrónica?

\begin{tabular}{|c|c|}
\hline $\begin{array}{ll}\text { OPINIONES POSITIVAS: } \\
\text { - inmediata } & \text { - accesible } \\
\text { - útil } & \text { - rápida } \\
\text { - necesaria } & \text { - barata } \\
\text { - profesional } & \text { - completa } \\
\text { - gratis } & \text { - objetiva } \\
\text { - práctica } & \text { - fácil de usar } \\
\text { - ahorra tiempo } & \end{array}$ & $\begin{array}{l}\text { OPINIONES NEGATIVAS: } \\
\text { - poco fiable } \\
\text { - está politizada } \\
\text { - sometida a intereses empresariales } \\
\text { - publica rumores } \\
\text { - profundiza poco } \\
\text { - la publicidad es molesta } \\
\text { - saturación informativa }\end{array}$ \\
\hline $\begin{array}{l}\text { AGENTE EDUCADOR: } \\
\text { - buena herramienta educativa } \\
\text { - la uso en mis clases } \\
\text { - la puedes usar en algunas asignaturas }\end{array}$ & $\begin{array}{ll}\text { NO LA USA: } & \\
\text { - no la uso } & \text { - la uso poco } \\
\text { - no la conozco } & \text { - solo leo prensa papel } \\
\text { - no la leo } & \end{array}$ \\
\hline
\end{tabular}

Al analizar los resultados (Gráfica 2) ${ }^{1}$ deducimos que, respecto a la opinión del profesorado sobre la prensa electrónica, predominan las percepciones positivas, tanto en Primaria (63\%) como en Secundaria Obligatoria (64\%). Así pues, las principales ventajas que los docentes asocian a este medio son la gratuidad, la facilidad de acceso y la rapidez con la que se divulgan las noticias. Además de que es fácil de usar, completa y variada respecto a sus contenidos, y permite ahorrar tiempo. Por el contrario, el 12\% del profesorado de Primaria y el 13\% de Secundaria han respondido con comentarios negativos; de manera que critican la falta de objetividad, el hecho de que la ciberprensa está influenciada por intereses políticos y empresariales, que profundiza poco, que la publicidad online es molesta y que publica rumores. Cabe mencionar que las peores percepciones se han detectado en el grupo de los profesores hombres de Primaria.

Un reducido sector del profesorado, el 7\% de Primaria y el 4\% de Secundaria, ha comentado la utilidad de la prensa digital como un recurso didáctico en el aula, calificándola como una buena herramienta educativa que usa habitualmente en sus clases. Atendiendo a las variables, las maestras de Primaria son las que destacan en este sentido.

Finalmente, figura el grupo de docentes que no usa o no conoce la prensa digital. En concreto, se trata del 9\% del profesorado de Primaria y el 5\% de

1. En las gráficas los porcentajes totales pueden sobrepasar el 100\% debido a que en las preguntas del cuestionario el profesorado podía aportar más de una respuesta. 
ACTITUDES DOCENTES HACIA LA INTEGRACIÓN CURRICULAR DE LA PRENSA DIGITAL EN LOS CENTROS ESCOLARES DE EDUCACIÓN OBLIGATORIA

Secundaria. Predominan en esta categoría los sujetos de Educación Primaria, en los grupos de las profesoras, el profesorado que tiene de 36 a 45 años y de colegios públicos. En cambio, ningún docente joven de Educación Secundaria, menor de 35 años, ha señalado que no usa o no conoce la prensa digital.

GRÁFICA 2

Porcentajes correspondientes a la pregunta: ¿Qué opina de la prensa electrónica?

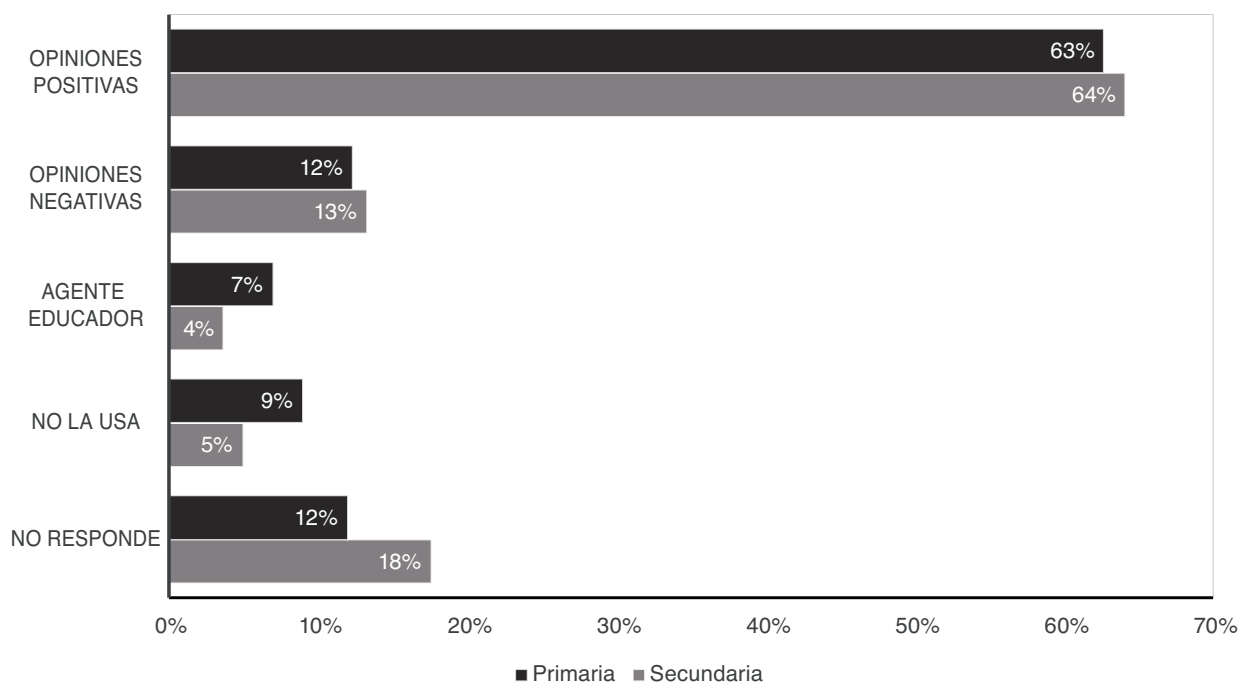

\section{2. ¿Cómo mejoraría la prensa digital?}

Según los resultados derivados del análisis, el profesorado de Educación Obligatoria ha sugerido medidas para mejorar la calidad de la ciberprensa. Observamos que entre las propuestas predominan las siguientes: «emplear una publicidad menos invasiva", "más veracidad", "más objetividad", "una prensa digital más independiente de los poderes políticos y económicos", "implantar sistemas que le permitan al lector confirmar la información con las fuentes originales», "que todos los contenidos sean gratuitos", "más artículos de opinión", "más textos de investigación", "contratar a periodistas más expertos", "más textos de análisis", "textos más extensos», "más soporte audiovisual», "más aplicaciones para el móvil», etc.

\subsection{En su labor docente, ¿en qué materias usaría la prensa electrónica?}

Con esta pregunta pretendemos conocer en qué asignatura/s utilizarían los profesores la prensa digital como una herramienta educativa. Una vez leídos y transcritos todos los textos las respuestas se codificaron y distribuyeron empleando 
las siguientes etiquetas: 1) Ninguna asignatura; 2) Todas las asignaturas; 3) Asignaturas de Letras; 4) Asignaturas de Ciencias; 5) Otras asignaturas (Cuadro 3).

\section{CUADRO 3}

Codificación de las unidades de significación en la pregunta:

En su labor docente, ¿en qué materias usaría la prensa electrónica?

\begin{tabular}{|l|l|}
\hline EN NINGUNA ASIGNATURA & EN TODAS LAS ASIGNATURAS \\
\hline ASIGNATURAS DE LETRAS: & ASIGNATURAS DE CIENCIAS: \\
- Lengua y Literatura & - Matemáticas \\
- Historia & - Ciencias Sociales y Naturales \\
- Latín & - Geografía \\
- Filosofía & - Física y Química \\
- Inglés & - Biología \\
\hline OTRAS ASIGNATURAS: & NO RESPONDE \\
- Educación Física - Música & \\
- Religión - Tecnología & \\
- Plástica & \\
\hline
\end{tabular}

En base a los datos obtenidos (Gráfica 3), podemos concluir que son muy pocos los docentes, menos de un $4 \%$ en ambas especialidades académicas, que no usarían la prensa digital en ninguna asignatura. Mientras que un porcentaje mayor, que ronda el 20\% de los docentes, considera que la ciberprensa se puede emplear en todas las materias del currículum. Por su parte, la mayoría del profesorado, que supone el $61 \%$ de Primaria y el $43 \%$ de Secundaria Obligatoria, apuesta por emplear las publicaciones electrónicas en asignaturas de Ciencias, como es el caso de Matemáticas, Ciencias Sociales, Ciencias Naturales y Física. También la mayoría, el $53 \%$ de los docentes de Primaria y el 46\% de Secundaria, manifiesta que las usaría en materias específicas de Letras como Lengua, Literatura e Inglés. En ambos casos, predominan las respuestas de los profesores varones de Educación Primaria.

Por último, figuran aquellos docentes, mayoritariamente de Secundaria (15\%) en los grupos de hombres, de sujetos que tienen entre 36 y 45 años y de colegios concertados, que también usarían la prensa digital en otras asignaturas distintas a las anteriormente citadas como son Educación Física, Música, Religión y Ciudadanía.

\section{4. ¿Y para qué tipo de actividades usaría la prensa electrónica?}

Para llevar a cabo la codificación de las respuestas se creó la categoría 'Actividades', referida a los trabajos con la prensa digital que pueden aplicarse a cualquier materia, con una serie de subcategorías, que se corresponden con las distintas asignaturas del currículo, tal y como puede apreciarse en el Cuadro 4. 
ACTITUDES DOCENTES HACIA LA INTEGRACIÓN CURRICULAR DE LA PRENSA DIGITAL EN LOS CENTROS ESCOLARES DE EDUCACIÓN OBLIGATORIA

\section{GRÁFICA 3}

Porcentajes correspondientes a la pregunta:

En su labor docente, ¿en qué materias usaría la prensa electrónica?

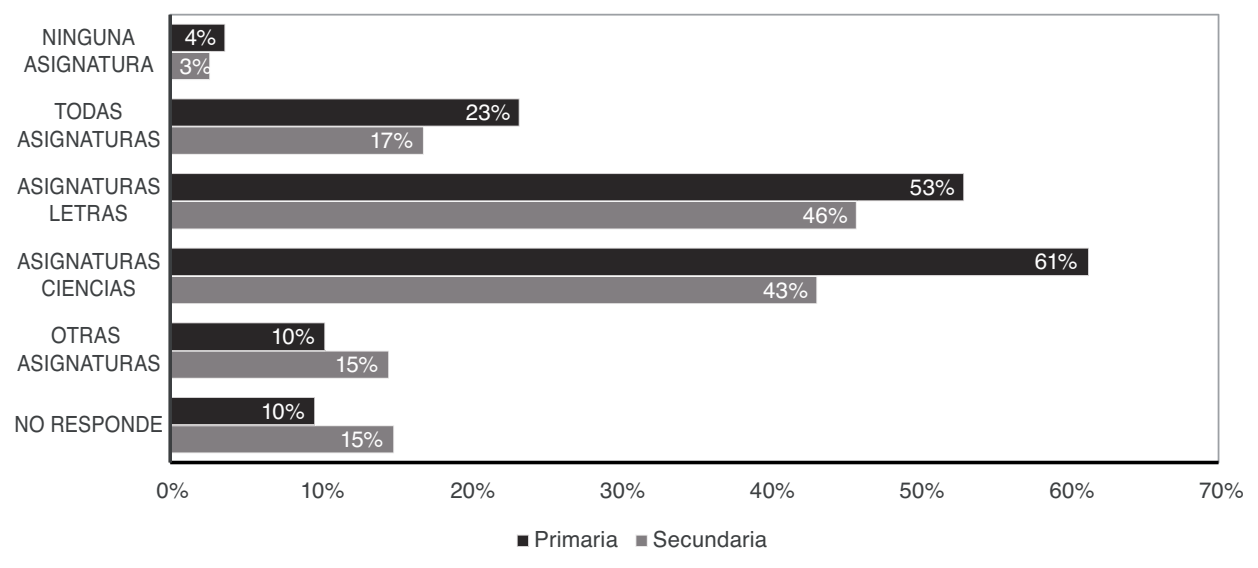

CUADRO 4

Codificación de las unidades de significación en la pregunta 4: ¿Y para qué tipo de actividades usaría la prensa electrónica?

\begin{tabular}{|l|l|}
\hline DE CUALQUIER ASIGNATURA: & ACTIVIDADES DE LETRAS: \\
- debates sobre el temario & - comentarios de textos \\
- buscar información en la Red & - estudiar los textos periodísticos \\
- leer las noticias del día & - ejercicios de gramática y ortografía \\
- redactar noticias propias & - comprensión lectora y expresión oral \\
- comenzar una clase & - aprender vocabulario \\
- hacer monográficos & - leer noticias en inglés \\
- complemento al libro de texto & - hacer traducciones \\
\hline ACTIVIDADES DE CIENCIAS: & OTRAS ACTIVIDADES: \\
- análisis de gráficas & - leer críticas deportivas \\
- leer noticias con cifras y porcentajes & - leer críticas de conciertos \\
- resolver problemas estadísticos & - usar la prensa digital como tecnología \\
- analizar diagramas publicados en noticias & - leer noticias sobre avances tecnológicos \\
- leer noticias de política y geografía & - comentar noticias sobre valores éticos \\
- leer noticias sobre medio ambiente & NO RESPONDE \\
- hacer investigaciones en equipo & \\
\hline
\end{tabular}

Los resultados (Gráfica 4) revelan que la mayoría del profesorado, casi el 80\%, tanto en Primaria como en Secundaria, y especialmente en el caso de las mujeres 
y los docentes más jóvenes, sugiere actividades para trabajar con la prensa digital en cualquier asignatura: debates, búsqueda de información, leer las noticias del día, hacer murales sobre la materia, introducir una clase, complemento al libro de texto, etc. Por su parte, alrededor del 12\% de los docentes, sobre todo los hombres, aportan actividades con los cibermedios que son específicas para las asignaturas de Letras como pueden ser comentarios de texto, ejercicios de gramática y ortografía, estudiar los textos periodísticos, aprender vocabulario, leer noticias en inglés, hacer traducciones, etc. En un porcentaje similar, que es mayor en el caso de los centros públicos, los profesores y las profesoras también proponen ejercicios para trabajar con la prensa digital en materias de Ciencias: el análisis de gráficas, la lectura de noticias científicas, leer noticias con cifras y porcentajes, leer noticias sobre geografía y medio ambiente, etc. Finalmente, una minoría de sujetos, el 1\% en Primaria y el 3\% en Secundaria, plantea actividades en las que los diarios digitales se pueden emplear como una herramienta didáctica en asignaturas distintas a las anteriormente citadas: lectura de críticas musicales (Música), usar la prensa digital como tecnología (Informática) y trabajos colaborativos para fomentar valores cívicos (Ciudadanía).

\section{GRÁFICA 4}

Porcentajes correspondientes a la pregunta:

¿Y para qué tipo de actividades usaría la prensa electrónica?

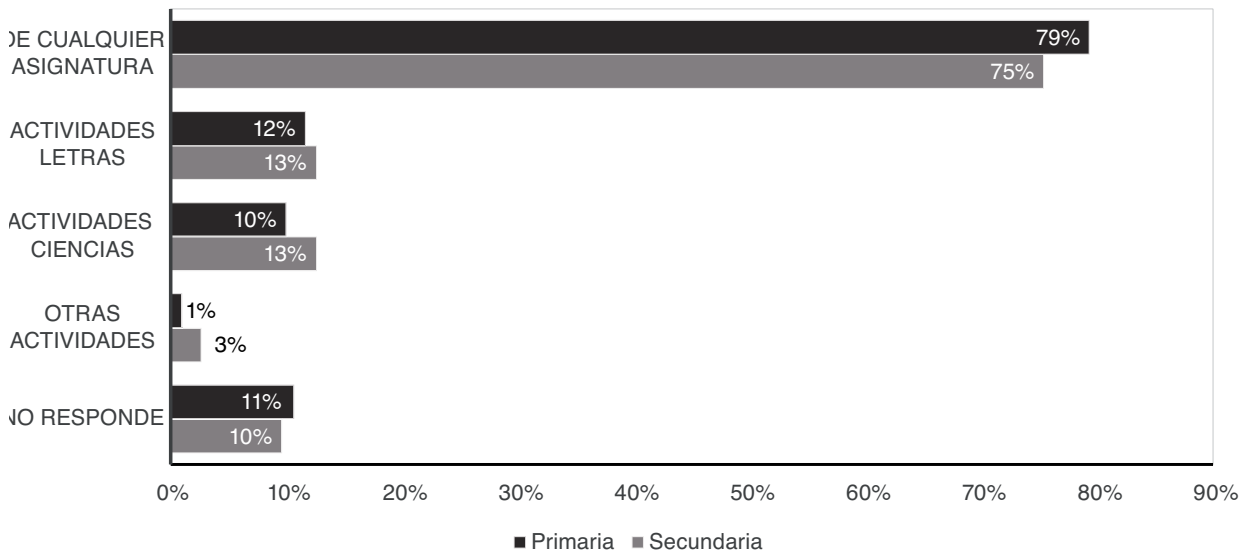

\section{5. ¿Cuáles son las capacidades que presentan los profesores a la bora de educar en medios de comunicación digitales?}

En relación a esta pregunta, los docentes han señalado su capacidad didáctica o pedagógica, a las que se suman otras como la capacidad para seleccionar y emplear con criterio los contenidos de la prensa online, el manejo de textos, el espíritu autodidacta, el conocimiento del lenguaje, que son orientadores, las ganas 
ACTITUDES DOCENTES HACIA LA INTEGRACIÓN CURRICULAR DE LA PRENSA DIGITAL EN LOS CENTROS ESCOLARES DE EDUCACIÓN OBLIGATORIA

de innovar, el dominio de las situaciones que se producen en clase, la capacidad para interpretar las noticias, la teatralidad, etc. Atendiendo a las variables, cabe destacar que los hombres y los maestros más jóvenes de Primaria son los que en mayor medida defienden estas habilidades del profesorado.

\section{6. ¿Cuáles son las carencias que presentan los profesores a la bora de educar en medios de comunicación digitales?}

Respecto a las carencias del profesorado a la hora de integrar y manejar los medios de comunicación online como herramienta didáctica, las opiniones se han codificado en tres etiquetas (Cuadro 5): 1) Carencias tecnológicas. Incluye las respuestas relacionadas con la escasez o falta de recursos tecnológicos del centro escolar; 2) Carencias formativas. Referida a la falta de conocimiento e información de los profesores; 3) Otras carencias. En alusión a las carencias docentes en un sentido más genérico.

\section{CUADRO 5}

Codificación de las unidades de significación en la pregunta: ¿Cuáles son las carencias que presentan los profesores a la hora de educar en medios de comunicación digitales?

\begin{tabular}{|l|l|}
\hline TECNOLÓGICAS: & $\begin{array}{l}\text { FORMATIVAS: } \\
\text { - falta formación sobre prensa digital }\end{array}$ \\
- falla la conexión a Internet & - alfabetización mediática \\
- no hay pizarra digital & - desconocimiento de sus aplicaciones docentes \\
- no hay suficientes ordenadores & - no sabemos hacer páginas web \\
- no hay mantenimiento de los equipos & - cursos de competencia digital experta \\
- los equipos fallan continuamente & NO RESPONDE \\
\hline $\begin{array}{l}\text { OTRAS CARENCIAS: } \\
\text { - falta de tiempo y de oportunidades }\end{array}$ & \\
- sin apoyo de la Administración & \\
- faltan interés y motivación & \\
- faltan ganas de innovar & \\
- falta de uso y de experiencia & \\
- no hay un programa para su uso en el centro & \\
- no se practica con los niños pequeños & \\
\hline
\end{tabular}

Según los resultados (Gráfica 5), destacan aquellas carencias que están relacionadas con la equipación del centro escolar y con la propia formación del profesorado. Los docentes señalan que sus centros, sobre todo las escuelas públicas de Primaria, no cuentan con los recursos tecnológicos necesarios para emplear correctamente la ciberprensa en la clase, pues falla la conexión a Internet, los 
ordenadores son muy antiguos o carecen de pizarra digital. Por su parte, las carencias formativas se centran en aspectos como la necesidad de un mayor conocimiento en prensa digital, programas de alfabetización mediática y destreza con las TIC. Estas percepciones que acabamos de comentar son más visibles en Primaria, en los grupos de mujeres y de colegios concertados.

Finalmente, los docentes también hacen referencia a la falta de interés y de motivación, la falta de tiempo y de práctica; y solicitan un programa para el uso específico de la prensa digital con los alumnos en el centro escolar. Estas últimas apreciaciones son más patentes en los profesores más jóvenes de Educación Secundaria.

\section{GRÁFICA 5}

Porcentajes correspondientes a la pregunta: ¿Cuáles son las carencias que presentan los profesores a la hora de educar en medios de comunicación digitales?

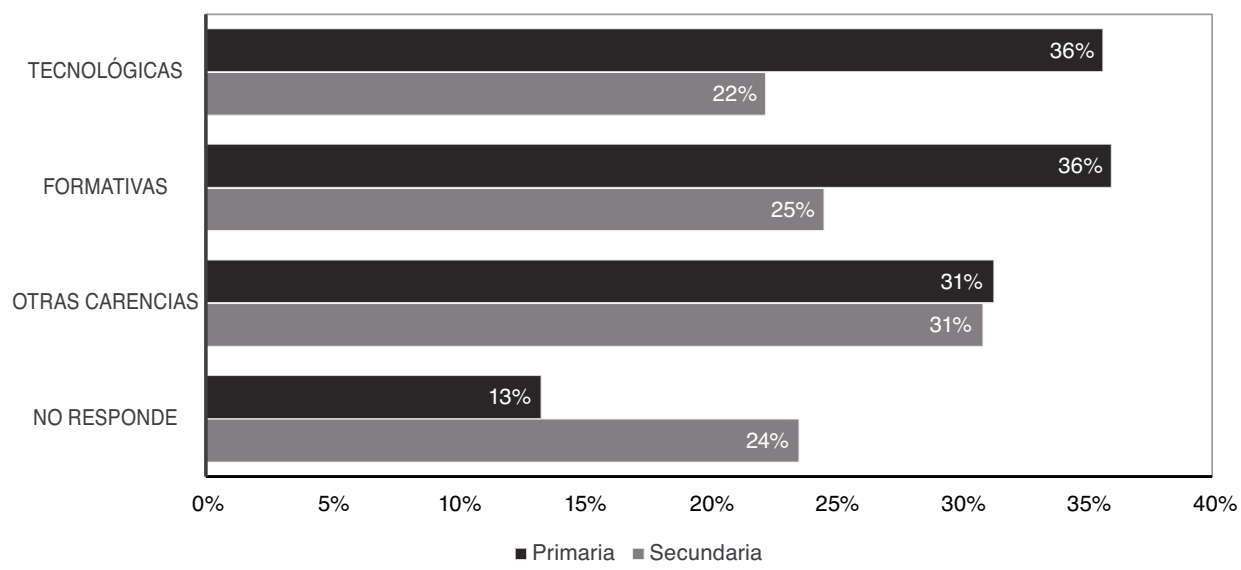

4.7. Según su opinión, ¿cómo influye el ambiente familiar en el uso que los niños y los adolescentes hacen de la prensa electrónica?

Después de haber leído todas las respuestas y observar cómo se repetían determinadas temáticas y enfoques, las unidades significativas relativas a esta pregunta se han codificado atendiendo a seis categorías, tal y como se muestra en el Cuadro 6. 
ACTITUDES DOCENTES HACIA LA INTEGRACIÓN CURRICULAR DE LA PRENSA DIGITAL EN LOS CENTROS ESCOLARES DE EDUCACIÓN OBLIGATORIA

\section{CuAdro 6}

Codificación de las unidades de significación en la pregunta: Según su opinión, ¿cómo influye el ambiente familiar en el uso que los niños y los adolescentes

hacen de la prensa electrónica?

\begin{tabular}{|c|c|}
\hline $\begin{array}{l}\text { FACTORES DEMOGRÁFICOS: } \\
\text { - ambiente familiar } \\
\text { - uso continuado de la prensa digital } \\
\text { - nivel social y nivel cultural } \\
\text { - tener ordenadores en casa } \\
\text { - tendencia ideológica }\end{array}$ & $\begin{array}{l}\text { OPINIONES NEGATIVAS: } \\
\text { - la familia no lee prensa digital } \\
\text { - se informan mediante las redes sociales } \\
\text { - en casa sólo leen prensa deportiva o rosa } \\
\text { - en casa sólo se ve la televisión } \\
\text { - no tienen ordenador en casa: brecha digital } \\
\text { - les falta formación crítica } \\
\text { - no saben filtrar las noticias }\end{array}$ \\
\hline $\begin{array}{l}\text { CONTROL PARENTAL: } \\
\text { - controlar el acceso de los niños a Internet } \\
\text { - no controlan lo que ven los niños en la Red } \\
\text { - acceso solo a contenidos apropiados }\end{array}$ & $\begin{array}{l}\text { LOS NIÑOS IMITAN A LOS PADRES: } \\
\text { - los niños hacen lo que ven en casa } \\
\text { - por imitación de las conductas de sus padres } \\
\text { - las familias lectoras hacen hijos lectores } \\
\text { - de padres lectores, salen niños lectores }\end{array}$ \\
\hline $\begin{array}{l}\text { ROL EDUCADOR DE LA FAMILIA: } \\
\text { - dialogar sobre su uso responsable } \\
\text { - fomentando su uso desde que son pequeños } \\
\text { - formar a las familias en la prensa digital } \\
\text { - crear un hábito de lectura de prensa digital } \\
\text { - enseñando a distinguir lo real de lo falso } \\
\text { - analizar las noticias en casa }\end{array}$ & NO RESPONDE \\
\hline
\end{tabular}

Se aprecia (Gráfica 6) que al responder a esta pregunta un pequeño grupo de docentes, en torno al 8\%, han expuesto cuáles son los factores de los que depende la influencia familiar sobre el consumo que hacen los alumnos de las publicaciones electrónicas, como es el caso del ambiente en el hogar, el nivel social, la presencia de ordenadores en casa o la tendencia ideológica. También se han detectado opiniones negativas sobre esta influencia familiar pues, según afirma una media del $14 \%$ de los docentes, la familia no lee la prensa digital y prefiere informarse a través de las redes sociales y de la televisión, o bien sólo lee prensa deportiva o la prensa del corazón. Además, o no tienen ordenador en casa o los propios padres no saben hacer un buen uso de la prensa online.

Sin embargo, otro sector del profesorado, en torno al 16\% y preferentemente en el caso de los docentes más jóvenes de Secundaria, ha destacado el papel educador de la familia en lo que se refiere al uso responsable de los cibermedios, pues la familia puede enseñar a los hijos a usar la prensa de una manera consciente, fomentar en ellos el hábito de leer las publicaciones online, así como ayudarles a 
distinguir lo real de lo falso. De tal forma que «si los padres debaten, comentan y ayudan a los niños a interpretar el contenido mediático, pueden ayudarlos a comprender mejor los mensajes producidos por los medios» (Pereira, Pinto y Pereira, 2012: 93).

La categoría con mayor representación, en torno al 35\% de los docentes en ambas especialidades, ha sido aquella donde se considera que la influencia de la familia existe en la medida que los menores imitan a los mayores a la hora de consultar la prensa digital, heredando así determinados hábitos y costumbres.

Por último, una minoría opina que la influencia de las familias en el uso que hacen los alumnos de la prensa online está relacionada con el control de los padres sobre el acceso de sus hijos a Internet. Por variables, quienes más insisten en esta idea del control parental son los docentes de Primaria en los grupos de hombres, de los sujetos más jóvenes y de centros públicos.

\section{GRÁFICA 6}

Porcentajes correspondientes a la pregunta: Según su opinión, ¿cómo influye el ambiente familiar en el uso que los niños y los adolescentes hacen de la prensa electrónica?

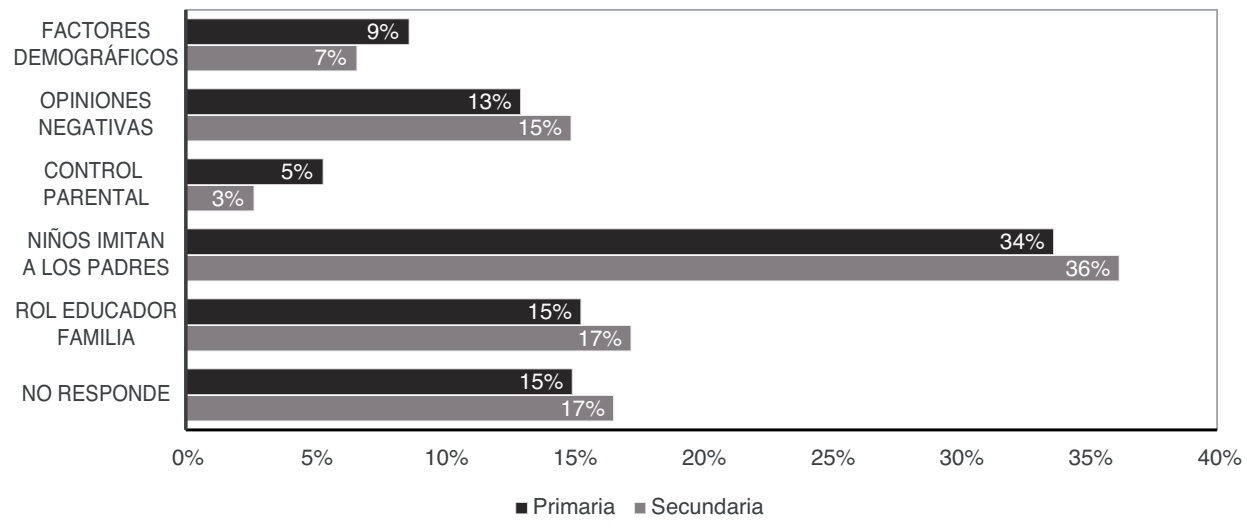

4.8. ¿Cómo puede influir el centro escolar en la utilización de la prensa digital por parte de los alumnos?

Con esta pregunta pretendemos conocer, según la percepción del profesorado, de qué forma afecta el centro escolar al uso que hacen los niños y los jóvenes de los medios de comunicación digitales, tanto dentro como fuera del ámbito educativo. Entre las respuestas se aprecian claramente ciertas opiniones que nos han permitido establecer seis categorías de codificación, que han quedado reflejadas en el siguiente Cuadro 7. 
ACTITUDES DOCENTES HACIA LA INTEGRACIÓN CURRICULAR DE LA PRENSA DIGITAL EN LOS CENTROS ESCOLARES DE EDUCACIÓN OBLIGATORIA

\section{CUADRO 7}

Codificación de las unidades de significación en la pregunta: ¿Cómo puede influir el centro escolar en la utilización de la prensa digital por parte de los alumnos?

\begin{tabular}{|l|l|}
\hline OPINIONES NEGATIVAS: & $\begin{array}{l}\text { FACETA EDUCATIVA DEL CENTRO: } \\
\text { - enseñar a interpretar y contratar las noticias } \\
\text { - no es un tema prioritario }\end{array}$ \\
$\begin{array}{l}\text { - nada porque no hay tiempo ni medios } \\
\text { - poco porque el programa no lo permite }\end{array}$ & $\begin{array}{l}\text { - enseñarle a pensar y buscar la verdad } \\
\text { - enseñar a distinguir la noticia de la opinión } \\
\text { - conociendo los riesgos } \\
\text { - aprendizaje colaborativo } \\
\text { - creando hábitos de lectura }\end{array}$ \\
\hline $\begin{array}{l}\text { RECURSOS TIC: } \\
\text { - con pizarras electrónicas y ordenadores } \\
\text { - suscribirse a kioscosde prensa digital } \\
\text { - facilitando una red estable de Internet }\end{array}$ & $\begin{array}{l}\text { CREACIÓN DE PRENSA ESCOLAR: } \\
\text { - elaborar un periódico digital escolar } \\
\text { - hacer una revista digital en el centro }\end{array}$ \\
\hline $\begin{array}{l}\text { EL ROL DEL PROFESOR: } \\
\text { - formar a los profesores en prensa digital } \\
\text { - los profesores deben enseñar a leer la prensa } \\
\text { - la influencia depende de los profesores }\end{array}$ & \begin{tabular}{l} 
NO RESPONDE \\
\hline
\end{tabular}
\end{tabular}

En base a las respuestas obtenidas (Gráfica 7), observamos que una minoría de los sujetos, en torno a un 5\% de los docentes, y en mayor medida los profesores de Primaria que tienen más de 46 años, considera que la influencia del centro escolar sobre el uso que hacen los alumnos de la prensa digital es negativa, pues no es un tema prioritario para la escuela, no hay ni tiempo ni medios o el programa no lo permite.

Sin embargo, la mayoría del profesorado de ambas especialidades, y preferentemente en Primaria, destaca el papel educador que tiene el centro en la relación que mantenga el estudiante con la prensa online, dado que pueden ser funciones de la escuela enseñar al estudiante a interpretar y contrastar las noticias, y que aprenda su uso con sentido propio. De hecho, los docentes sugieren interesantes ideas enfocadas a la integración y el uso de los cibermedios como un recurso docente: «realizar actividades lúdicas y extraescolares con la prensa digital que sean un complemento de las asignaturas", "crear hábitos de lectura", "acercar al alumno a la realidad mediante la prensa electrónica", "crear un taller de prensa", "Organizar concursos en torno a la prensa digital», "diseñar un reglamento de uso de la prensa digital", etc.

A pesar de ser una eficaz propuesta para educar en medios de comunicación digitales y familiarizarse con el uso de la prensa online, sólo el $4 \%$ del profesorado propone la elaboración de prensa digital escolar. Igualmente, también son 
muy pocos los docentes que destacan la importancia del profesor en relación a la influencia que tiene el centro escolar sobre el comportamiento de los alumnos con la ciberprensa. En este sentido, opinan que hay que formarlos para que estén cualificados y sepan seleccionar las noticias más apropiadas para sus clases entre aquellas que se publican en la prensa digital.

Por último, una minoría de docentes, en torno al 7\% en ambas especialidades, hace referencia a los recursos digitales con los que cuenta el centro escolar y considera que la infraestructura es un factor que condiciona el empleo de los cibermedios por parte de los alumnos; por lo que se hace preciso disponer de las herramientas necesarias para su uso didáctico.

\section{GRÁFICA 7}

Porcentajes correspondientes a la pregunta: ¿Cómo puede influir el centro escolar en la utilización de la prensa digital por parte de los alumnos?

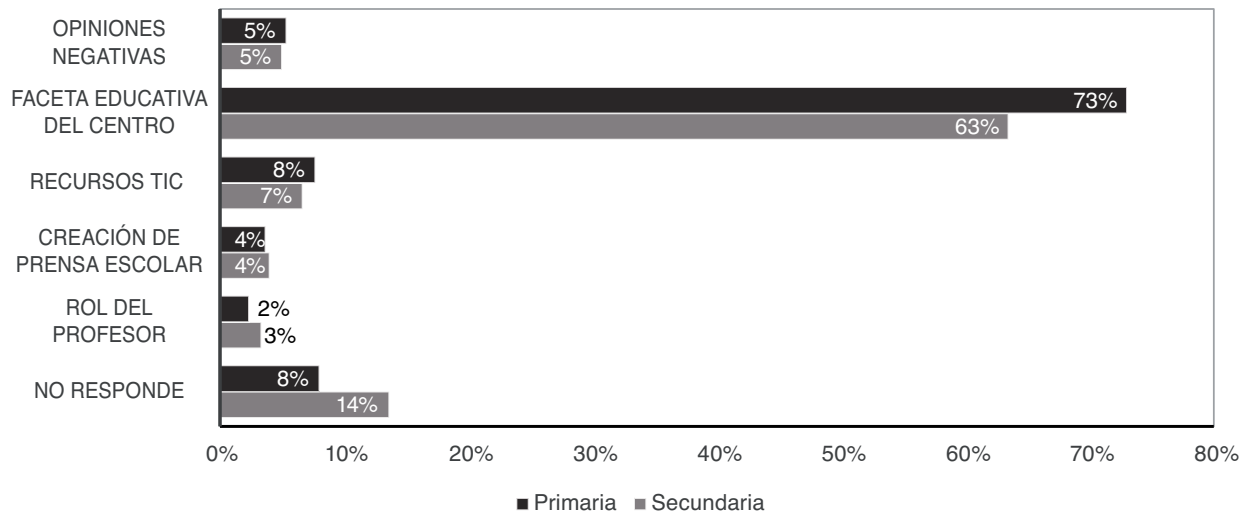

\section{CONCLUSIONES Y DISCUSIÓN}

Esta investigación se planteó con el objetivo de conocer las actitudes del profesorado de Educación Obligatoria hacia la integración escolar de las publicaciones online. Se detectó que la mayoría de los docentes tiene una opinión positiva sobre la prensa digital, pues les resulta completa, fácil de usar, económica y rápida. Aunque también manifiestan opiniones negativas como que es poco objetiva y está sometida a intereses políticos y empresariales. En este sentido, el profesorado propone mejoras tales como emplear una publicidad menos invasiva, más textos de opinión y reportajes de investigación, permitir que el lector pueda confirmar la información con las fuentes originales y mayor veracidad.

Atendiendo a las propuestas del profesorado, la prensa digital podría emplearse como herramienta educativa en prácticamente todas las asignaturas de la Educación Obligatoria. Respecto al tipo de actividades en las que estos docentes utilizarían la ciberprensa, son muchas y variadas, y entre ellas figuran: hacer debates, 
ACTITUDES DOCENTES HACIA LA INTEGRACIÓN CURRICULAR DE LA PRENSA DIGITAL EN LOS CENTROS ESCOLARES DE EDUCACIÓN OBLIGATORIA

introducción a una clase, estudiar los textos periodísticos, mejorar el vocabulario, leer noticias en inglés, leer noticias con cifras y gráficas, realizar trabajos colaborativos que fomenten los valores cívicos, etc.

Al ser preguntados sobre sus capacidades para educar en medios de comunicación digitales, el profesorado manifiesta que sus habilidades son muchas y valiosas, tales como el manejo de textos, el conocimiento del lenguaje, su capacidad crítica, que son orientadores o el espíritu autodidacta. Respecto a las carencias, destacan la escasez de recursos tecnológicos con los que cuentan sus centros escolares y la falta de planes de formación, siendo las profesoras de Primaria quienes se consideran menos preparadas para trabajar con la ciberprensa en el aula. De manera que, en la mayoría de los casos, solicitan ordenadores, conexión a Internet y pizarras digitales; y plantean la necesidad de programas de alfabetización mediática y destreza con las TIC.

Sobre el rol que desempeña el ambiente familiar en el uso que los niños y los jóvenes hacen de la prensa electrónica, en torno al 35\% del profesorado ha manifestado que los menores imitan a los mayores a la hora de consumir los medios de comunicación. Mientras que otros docentes, especialmente los más jóvenes en Secundaria Obligatoria, destacan el papel educador de la familia, pues puede fomentar entre los hijos una lectura responsable y ayudarles a distinguir entre las noticias reales y la información falsa. Por el contrario, un 14\% considera que esta influencia es negativa, pues la familia ve mucha televisión, pero lee poca prensa digital y prefiere consultar las redes sociales.

Por último, en relación a la influencia que ejerce el centro escolar sobre la utilización de la prensa digital por parte de los alumnos, la mayoría del profesorado, preferentemente en Primaria, destaca el papel educador de la escuela en este sentido, pues puede enseñar al estudiante a entender y contrastar las noticias en la Red, de modo que haga un uso responsable y beneficioso de estos medios.

En base a esta información, interpretamos que el profesorado contempla la prensa digital como un agente educador. Atendiendo al género, los resultados revelan que los profesores varones son más proclives al uso de la ciberprensa como herramienta educativa, coincidiendo así con el estudio de Suárez et al. (2012), donde se concluye que los hombres tienen en mayor consideración la integración escolar de los medios técnicos y digitales.

Igualmente, las percepciones de los docentes han permitido, por un lado, constatar su creatividad y competencia a la hora de plantear actividades educativas con la ciberprensa; y, por otro, detectar carencias ya identificadas en anteriores investigaciones (Cabero, 2000; Cuervo, Armenta, Martínez, López, y Lozoya, 2011; Pérez y Rodríguez, 2016; Sangrá, 2016), entre las que figuran la escasez de medios técnicos y de habilidades específicas para trabajar en el aula con las publicaciones digitales. Siendo ambos motivos factores de peso en el escaso empleo que los centros escolares consultados hacen de las publicaciones online.

Respecto a las implicaciones de la investigación, el diagnóstico de estas oportunidades y necesidades debe plantearse como un marco de referencia para la 
puesta en marcha de acciones educativas. En este sentido, se hace preciso un plan de alfabetización dirigido al profesorado. Y para ello, se propone la ejecución de programas similares a los que desarrollan organismos tales como la Asociación de Educación Periodística (Journalism Education Association, 2016) y la Sociedad Americana de Editores de Noticias (American Society of News Editors, 2016), en Estados Unidos, y el CLEMI (2016) en Francia, destinados a educar en medios y herramientas digitales a los docentes para que puedan explicar y emplear en sus clases conceptos y recursos periodísticos.

En relación al alumnado, conviene atender aquellas actividades en el aula que implican la lectura de noticias en Internet (Singer y Alexander, 2016; Kim, Hwang y Rho, 2016; Digital NIE, 2007). Así como la creación de prensa electrónica escolar (Mena, 2016; Trillo, 2003; Canavilhas, Merino-Arribas y Kroth, 2016) por tratarse de una actividad que permite implicar a toda la comunidad educativa aprovechando las ventajas didácticas de la "galaxia mediática" (Cabero y Aguaded, 2014: 70). En estos procesos, tal y como advierte Kumaravadivelu (2012), el estudiante invierte en su educación de manera significativa, aprendiendo a pensar en cómo se consumen las noticias de la Red y en cómo ser pensadores críticos para poder participar en las conversaciones que a diario plantean los medios de comunicación digitales (Hayt, 2015).

Una de las limitaciones de esta investigación es la obsolescencia de los datos, pues el continuo cambio que experimentan los recursos informativos en Internet puede variar las percepciones docentes. Así pues, la investigación debe ser revisada periódicamente y recurrir a un enfoque longitudinal para conocer si las opiniones de los profesores cambian o se mantienen.

Finalmente, se sugieren algunas prospectivas de investigación para futuros estudios. En este sentido, algunas opciones son el planteamiento y evaluación de modelos pedagógicos que fomenten la integración curricular y el empleo de los cibermedios en clase; así como realizar un estudio de caso que permita compartir con el profesorado y los alumnos la experiencia de trabajar en el aula con estas plataformas a partir de unas unidades didácticas diseñadas ad hoc. También, basándonos en una sugerencia de Ordóñez-Sierra (2001), comparar las percepciones del profesorado sobre la prensa digital en zonas urbanas y rurales para conocer las diferencias que pudieran existir entre ambos medios.

\section{REFERENCIAS BIBLIOGRÁFICAS}

Alonso, R.; Ospina, P. y Sánchez, P. A. (2014). Aulas prensa. Hacia una lectura crítica de artículos de opinión en la educación media. Actualidades Pedagógicas, 63, 99-116.

Amar, V. (2010). La educación en medios digitales de educación. Pixel-Bit, 36, 115-124.

American Society of News Editors. Youth journalism, 2016. Descargado el 2 de septiembre, 2016. http://asne.org/content.asp? $\mathrm{pl}=15 \& \mathrm{sl}=67 \&$ contentid $=67$.

Balestrini, M. (1999). Cómo se elabora un proyecto de investigación. Venezuela: Fotolito Quintana. 
ACTITUDES DOCENTES HACIA LA INTEGRACIÓN CURRICULAR DE LA PRENSA DIGITAL EN LOS CENTROS ESCOLARES DE EDUCACIÓN OBLIGATORIA

Ballesta, J. y Bernal, M. (2013). Comprender la actualidad en el aula y desarrollar el pensamiento crítico a través de la prensa digital. En Actas del II Congreso Internacional Educación Mediática y Competencia Digital (pp. 941-952). Descargado el 4 de mayo, 2015. http://www.uoc.edu/portal/es/symposia/congreso_ludoliteracy2013/programa/ ACTAS_EDUMED_2013.pdf.

Ballesta, J.; Cerezo, M. C. y Veas, A. (2014). Los jóvenes de educación secundaria ante el uso y consumo de las TIC. Revista Científica Electrónica de Educación y Comunicación en la Sociedad del Conocimiento, época II, año XIV, 1 (14), 22-40.

Buil, I.; Hernández, B. y Sesé, F. J. (2013). El papel de la prensa en la mejora del proceso de aprendizaje: Una aplicación en el EEES. Revista de Docencia Universitaria, 11 (3), 445-459.

Cabero, J. (Dir.) (2000). Uso de los medios audiovisuales, informáticos y las nuevas tecnologías en los centros andaluces. Sevilla: Kronos.

Cabero, J. y Aguaded, I. (2014). Avances y retos en la promoción de la innovación didáctica con las tecnologías emergentes e interactivas. Educar, 50 (número extraordinario), 67-83.

Cabero, J.; Llorente, M. C.; Puentes, A.; Marín, V. y Cruz, I. (2011). La competencia digital del profesorado: un estudio en la Pontificia Universidad Católica Madre y Maestra. Sevilla: Grupo de Investigación Didáctica de la Universidad de Sevilla.

Canavilhas, J.; Merino-Arribas, A. y Kroth, M. (2016). Impacto socioeducativo del periódico escolar: metaanálisis de tres proyectos de educación en Brasil, España y Portugal. EDMETTC. Revista de Educación Mediática y TIC, 5 (2), 51-70.

Cebrián Herreros, M. (2009). Comunicación interactiva en los cibermedios. Comunicar, 33 , 15-24. doi: 10.3916/c33-2009-02-001.

Celina, H. y Campo-Arias, A. (2005). Aproximación al uso del coeficiente alfa de Cronbach. Revista Colombiana de Psiquiatría, 34 (4), 572-580.

CLEMI. Éducation aux médias et à l'information, 2016. Descargado el 2 de septiembre, 2016. http://www.cndp.fr/crdp-lyon/-clemi-education-aux-medias-et-a-1-html.

Colás, P. (1998). El análisis cualitativo de datos. En L. Buendía, P. Colás y F. Hernández (Coords.). Métodos de investigación en psicopedagogía (pp. 287-311). Madrid: McGrawHill.

Cuervo, M. Á. A. V.; Armenta, J. A.; Martínez, M. L. U.; López, R. I. G. y Lozoya, S. V. M. (2011). Necesidades de capacitación de docentes de educación básica en el uso de las TIC. Pixel-Bit. Revista de Medios y Educación, 39, 211-223.

Delors, J. (1996). La educación encierra un tesoro. Informe a la unESCO de la Comisión Internacional sobre la educación para el siglo XXI. Madrid: Santillana-UNESCO.

Digital NIE (2017). A guide to using e-editions with NIE Programs. Arlington, USA: Newspaper Association of America.

Feijoo, B. (2015). La prensa digital y las redes sociales en la actividad 2.0 de los menores gallegos. Icono 14, 13 (1), 30-51. doi: 10.7195/ri14.v13i1.739.

Fernández, L. (2006). ¿Cómo analizar datos cualitativos? Butlletí LaRecerca, ficha 7. Descargado el 10 de marzo, 2015. http://www.ub.edu/ice/recerca/fitxes/fitxa7-cast.htm.

George, D. y Mallery, P. (2003). spss for Windows step by step: A simple guide and reference. Boston: Allyn y Bacon.

González Prieto, E. (2010). La prensa digital. Un recurso para educar en cibercomunicación con la actualidad mundial. En E. Said Hung (Ed.). Tics, comunicación y periodismo digital (pp. 51-71). Colombia: Ediciones Uninorte. 

EN LOS CENTROS ESCOLARES DE EDUCACIÓN OBLIGATORIA

Hayt, T. (2015, 30 de julio). News literacy lessons for social studies, English, science and math available (entrada de blog). Descargado de: http://jea.org/blog/2015/07/30/ news-literacy-lessons-for-social-studies-english-science-and-math-available/.

Journalism Education Association (2016). Resources for educators. Descargado el 2 de septiembre. http://jea.org/blog/category/resources-for-educators/.

Kerlinger, F. N. (1975). Investigación del comportamiento. México: Interamericana.

Kim, Y.; Hwang, E. y Rho, S. (2016). Twitter news-in-education platform for social, collaborative, and flipped learning. The Journal of Supercomputing, 1-19. doi: 10.1007/ s11227-016-1776-X.

Krippendorff, K. (1990). Metodología del análisis de contenido. Teoría y práctica. Barcelona: Paidós Ibérica.

Kumaravadivelu, B. (2012). Language teacher education for a global society: A modular model for knowing, analyzing, recognizing, doing, and seeing. New York: Routledge.

Little, C. (2014). How newspapers excel in educating our students. Association of America. Descargado el 2 de febrero, 2015. http://www.naa.org/News-and-Media/Blog/ How-newspapers-excel-in-educating-our-students.aspx.

López, L. y Aguaded, M. C. (2015). La docencia sobre alfabetización mediática en las facultades de Educación y Comunicación. Comunicar, 44, 187-195.

López Gil, K. S. (2016). Prácticas de lectura digital de estudiantes universitarios. Enseñanza E Teaching: Revista Interuniversitaria de Didáctica, 34 (1), 57-92. doi: 10.14201/ et20163415792.

Marí, V. M. (2006). Jóvenes, tecnologías y el lenguaje de los vínculos. Comunicar, 27, 113116.

Mejía, B. M. y Acero, E. (2015). Integración de medios de comunicación y TIC en el desarrollo de los espacios académicos de un grupo de docentes de la Universidad del Quindío. Colombia: Grupo de Investigación Comunicación, Cultura y Periodismo, Universidad del Quindío.

Mena, Y. P. (2016). Periódico digital de aula como recurso para estimular la lectura y la escritura en los estudiantes de quinto grado de la Escuela Básica 'Rafael Antonio Godoy'. Administración Educacional, 4, 21-22.

Moore, S. E. (2013). A Survey of Journalism Schools' Online Publication of Student Content. (Tesis doctoral). West Virginia, EE. UU.: West Virginia University.

Morduchowicz, R. (2012). La escuela, los medios y la cultura juvenil. [Con]textos, 1 (1), 21-24.

Ordóñez Sierra, R. (2001). Perspectivas sobre la participación de padres y profesores en los centros de Educación Primaria (Tesis doctoral). Sevilla: Universidad de Sevilla.

Pedrosa, J. (2010). La prensa digital en el aula. De los mass a los self media. Revista DIM, Didáctica, Innovación y Multimedia, 19. Descargado el 18 de mayo, 2015. dim.pangea. org/revistaDIM19/docs/jorgepedrosa.doc.

Pereira, S.; Pinto, M. y Pereira, L. (2012). Recursos para la alfabetización mediática: investigación y propuestas para niños. Comunicar, 39, 91-99. doi: 10.3916/C39-2012-02-09.

Pérez, A. y Rodríguez, M. J. (2016). Evaluación de las competencias digitales autopercibidas del profesorado de educación primaria en Castilla y León. Revista de Investigación Educativa, 34 (2), 399-415. doi: 10.6018/rie.34.2.215121.

Pinilla, R. (2008). Las posibilidades del periodismo digital como fuente de material didáctico para el aula de ELE. En S. Pastor Cesteros y S. Roca Marín (Coords.). La evaluación en el aprendizaje y la enseñanza del español como lengua extranjera/segunda lengua (pp. 489-496). Alicante: Universidad de Alicante. 
ACTITUDES DOCENTES HACIA LA INTEGRACIÓN CURRICULAR DE LA PRENSA DIGITAL EN LOS CENTROS ESCOLARES DE EDUCACIÓN OBLIGATORIA

Player-Koro, C. (2013). Hype, hope and ICT in teacher education: a Bernsteinian perspective. Learning, Media and Technology, 38 (1), 26-40.

Prensky, M. (2001). Digital natives, digital immigrants. University Press, 9 (5), 1-6.

Prensky, M. (2014). En Fominaya, C. Marc Prensky: «Los periodistas de hoy deberían eliminar las clases magistrales». ABC. Descargado el 20 de noviembre, 2014. https://goo.gl/ FEkHvO.

Ramírez, E.; Cañedo, I. y Clemente, M. (2012). Las actitudes y creencias de los profesores de secundaria sobre el uso de Internet en sus clases. Comunicar, 38, 147-155. doi: 10.3916/C38-2012-03-06.

Rodríguez, G.; Gil, J. y García, E. (1997). Metodología de la investigación cualitativa. Málaga: Aljibe.

Rubin, H. J y Rubin, I. S. (1995). Qualitative interviewing. The art of hearing data. Thousand Oaks, CA: Sage.

Said, E. (Ed.). (2010). Tic's, comunicación y periodismo digital (pp. 247-257). Colombia: Ediciones Uninorte.

Sangrá, L. M. (2016). La nueva brecha digital docente. El futuro de las nuevas tecnologías en Primaria desde la formación del profesorado. Revista Electrónica Interuniversitaria de Formación del Profesorado, 19 (2), 301-313. doi: 10.6018/reifop.19.2.189561.

Sierra, R. (1994). Técnicas de investigación Social. Teorías y ejercicios. Madrid: Paraninfo.

Singer, L. M. y Alexander, P. A. (2017). Reading Across Mediums: Effects of Reading Digital and Print Texts on Comprehension and Calibration. The Journal of Experimental Education, 85 (1), 155-172.

Snyder, S. (2016). Teachers' perceptions of digital citizenship development in middle school students using social media and global collaborative projects (Tesis doctoral). Minneapolis, EE. UU.: Walden University.

Spanhel, D. (2011). Un enfoque para integrar la educación en medios, en la instrucción y en el día a día de la escuela secundaria. Enseñanza \& Teaching: Revista Interuniversitaria de Didáctica, 29 (1), 181-190.

Suárez, J. M.; Almerich, G.; Díaz, I. y Fernández, R. (2012). Competencias del profesorado en las Tic. Influencia de factores personales y contextuales. Universitas Psychologica, 11 (1), 293-309.

Suriá, R. (2011). Percepción del profesorado sobre su capacitación en el uso de las Tic como instrumento de apoyo para la integración del alumnado con discapacidad. Profesorado, 15 (2), 299-314.

Trillo, M. (2003). The media in the classroom-a digital newspaper proposal: www. paraninfo. com. Educational Media International, 40 (3-4), 269-276.

UNESCO (2008). Estándares de competencias en TIC para docentes. Londres: Informe UNESCO. Descargado el 5 de febrero, 2014. http://www.oei.es/tic/UNESCOEstandaresDocentes. pdf. 